\title{
DIAGNÓSTICOS DE ENFERMAGEM MAIS PREVALENTES À PACIENTES EM PERIOPERATÓRIO DE NEOPLASIA DE CABEÇA E PESCOÇO
}

\section{NURSING DIAGNOSES MORE PREVALENT TO PATIENTS IN PERIOPERATORY NEOPLASIA OF HEAD AND NECK}

\author{
Letícia Gomes de Oliveira ${ }^{1} *$ Fernando Conceição de Lima $^{2} *$ Fabrício Gabriel Freitas Lima ${ }^{3} *$ \\ Thalyta Mariany Rêgo Lopes Ueno ${ }^{4} *$ Wagner Felipe dos Santos Neves ${ }^{5}$ Clarissa Porfírio Mendes ${ }^{6}$
}

\section{RESUMO}

Objetivo: Descrever a elaboração de uma tecnologia sobre diagnósticos de enfermagem mais prevalentes, segundo a taxonomia da NANDA International Inc. (NANDA-I), em pacientes perioperatório de cabeça e pescoço. Método: Estudo descritivo observacional, com abordagem qualitativa, do tipo Relato de Experiência, que descreve a atividade de residentes de Enfermagem no processo de elaboração de um produto de consulta adaptado sobre diagnósticos de enfermagem mais prevalentes em pacientes perioperatório de cirurgia de cabeça e pescoço. Resultados: O informativo foi dividido em domínios, conforme o exemplar NANDA-I, os tópicos foram definidos a partir de um diagnóstico situacional. Os itens que entraram para o conteúdo do impresso foram: I) Promoção da saúde, II) Nutrição III) Eliminação e Troca, IV) Atividade e repouso, V) Percepção/Cognição, VI) Auto-percepção, VII) papeis e relacionamento, VIII) Enfrentamento / tolerância ao estresse, IX) Segurança/proteção e X) Conforto. No final do impresso, fora inserido um $Q R$ code, com um link direto para o NANDA-I (2018-2021). Considerações finais: O estabelecimento de diagnósticos de enfermagem perpassa por um processo crítico, reflexivo que demanda conhecimento com base científica dos profissionais, que impactam na qualidade de vida que os usuários terão. A elaboração do produto sobre os principais diagnósticos na clínica de cabeça e pescoço do referido hospital, pelos residentes, demonstrou-se como uma estratégia de enorme potencial para orientação e organização das ações e serviços da enfermagem.

Palavras-chave: Diagnóstico de Enfermagem. Enfermagem. Neoplasia de Cabeça Pescoço. Neoplasias. Cuidados de Enfermagem.

\begin{abstract}
Objective: To describe the development of a technology on nursing diagnoses more prevalent, according to the taxonomy of NANDA International Inc. (NANDA-I) in head and neck perioperative patients. Method: Descriptive observational study, with qualitative approach, of the type Experience Report, which describes the activity of nursing residents in the process of elaboration of an adapted consultation product on nursing diagnoses more prevalent in perioperative patients of head and neck surgery. Results: The information was divided into domains, according to the NANDA-I copy, the topics were defined from a situational diagnosis. The items that entered the contents of the form were: I) Health promotion, II) Nutrition III) Elimination and Exchange, IV) Activity and rest, V) Perception/Cognition, VI) Self-perception, VII) roles and relationship, VIII) Coping / stress tolerance, IX) Safety/protection and X) Comfort. At the end of the form, a QR code was inserted, with a direct link to the NANDA-I (2018-2021). Final considerations: The establishment of nursing diagnoses permeates a critical, reflective process that demands knowledge based on the scientific basis of professionals, which impact on the quality of life that users will have. The elaboration of the product on the main diagnoses in the head and neck clinic of the referred hospital, by the residents, proved to be a strategy of enormous potential for guidance and organization of nursing actions and services.

Keywords: Nursing Diagnosis. Nursing. Head and Neck Neoplasms. Neoplasms. Nursing Care.

\footnotetext{
${ }^{1}$ Residente de Neurologia no Centro Universitário do Estado do Pará (CESUPA), Belém (PA), Brasil. Orcid: https://orcid.org/00000002-8830-728X

${ }^{2}$ Enfermeiro, Residente em Centro de Terapia Intensiva na Universidade do Estado do Pará (UEPA), Belém (PA), Brasil. Orcid: https://orcid.org/0000-0002-9418-3711

${ }^{3}$ Enfermeiro pela Universidade da Amazônia (UNAMA), Belém (PA), Brasil. Orcid: https://orcid.org/0000-0002-0686-733X

${ }^{4}$ Doutoranda em Biologia parasitária na Amazônia, professora na Universidade do Estado do Amazonas (UEA), Manaus (AM), Brasil. Orcid: https://orcid.org/0000-0002-3991-7022

${ }^{5}$ Enfermeiro, membro do Grupo de Estudos da reabilitação do Paciente Oncológico Cirúrgico da EERP/USP. Belém (PA), Brasil. Orcid: https://orcid.org/0000-0001-9989-7273

${ }^{6}$ Enfermeira. Mestre em Enfermagem e coordenadora da Residência uniprofissional no Holpital Ophir Loyola (HOL), Belém (PA), Brasil. Orcid: https://orcid.org/0000-0001-6488-718X
} 


\section{INTRODUÇÃO}

O termo coletivo câncer de cabeça e pescoço (CCP) é usado para definir um grupo de neoplasias heterogêneas que acometem o trato aero digestivo superior, compreendendo a $40 \%$ da cavidade bucal, como gengivas, palato, língua, soalho da língua, $15 \%$ na faringe, compreendendo a orofaringe, nasofaringe e hipofaringe e $25 \%$ acomete a laringe ${ }^{(1,2,3)}$.

O CCP alcança preocupantes $600 \mathrm{mil}$ casos novos a cada ano em todo o mundo e no Brasil é responsável por acometer 1,7\% da população, estando entre os dez tipos mais comuns e frequentes de câncer ${ }^{(4)}$. Estes tipos de tumores possuem origens multifatoriais, sendo que $80 \%$ de sua ocorrência está relacionada fatores ambientais, como estilo de vida, tabagismo, etilismo, Papiloma vírus humano, além dos fatores genéticos ${ }^{(5)}$.

O CCP acomete regiões anatômicas que são responsáveis por funções básicas como respiração, deglutição e comunicação verbal. O carcinoma de células escamosas é o tipo histológico predominante. Além disso, o próprio tratamento desses tipos de câncer pode prejudiciais para uma boa qualidade de $\operatorname{vida}^{(1,3)}$.

As principais complicações que o câncer de cabeça e pescoço pode ocasionar nas pessoas são: dificuldades na mastigação, disfagia, aspiração, alterações na fala e mudanças estéticas que comprometem negativamente os aspectos físicos e psicossociais desses pacientes ${ }^{(5,6)}$.

Por este motivo, a Resolução do Conselho Federal de Enfermagem (COFEN) $358 / 2009$ em seu art. $1^{\circ}$ O Processo de Enfermagem (PE) dispõe que o PE deve ser realizado, de modo deliberado e sistemático, em todos os ambientes, públicos ou privados, em que ocorre o cuidado profissional de Enfermagem. Esta é uma atividade privativa do enfermeiro, obedecendo a um encadeamento de cinco etapas entre elas o Diagnósticos de Enfermagem (DE) que fundamenta a prática assistencial atendendo as necessidades biopsicossociais ${ }^{(7,8)}$.

$\mathrm{O}$ enfermeiro assume um papel de extrema importância na assistência, e encontra no PE um subsídio para organizar o seu trabalho, que é desenvolvido em cinco etapas, sendo investigação, diagnóstico, planejamento, implementação e avaliação. Estabelece-se os DE a partir da identificação dos problemas do usuário, tornando o profissional de enfermagem capaz de compreender a pluralidade de cada ser e alcançar as prioridades, otimizando o cuidado e qualidade da assistência.

Reiterando que os enfermeiros são responsáveis por diagnosticar problemas de saúde, estados de risco e disposição para a promoção da saúde ${ }^{(9)}$. O Enfermeiro sendo parte integrante da equipe multiprofissional deve prestar atendimento avaliando e aplicando as normatizações e direcionamentos 
para benefício do seu serviço, considerando também as demandas e contextos sociais que estes indivíduos estão inseridos.

Nesse sentido, evidencia-se a relevância deste estudo por acreditar que o DE propicia uma interpretação fidedigna das necessidades dos usuários, bem como o agrupamento das informações colidas, que são essenciais para a decisão direcionada, na perspectiva de alcançar os resultados esperados mediante as melhores intervenções $^{(10)}$. Assim, com o presente estudo objetivou descrever a elaboração de uma tecnologia sobre diagnósticos de enfermagem mais prevalentes, segundo a taxonomia da NANDA International Inc. (NANDA-I), em pacientes perioperatório de cabeça e pescoço.

\section{MÉTODOS}

Trata-se de um estudo descritivo observacional, com abordagem qualitativa, do tipo relato de experiência, que descreve a atividade de residentes de um Programa de um hospital de referência em oncologia na cidade de Belém, no Estado do Pará no processo de elaboração de um produto tecnológico-assistencial sobre diagnósticos de enfermagem identificados em pacientes internados na clínica de cabeça e pescoço.

A referida Clínica tem um perfil de atendimento voltado para atender pacientes no perioperatório de cirurgias de cabeça e pescoço, como câncer de boca, orofaringe, nasofaringe, espaços parafaríngeos, nasais e de seios paranasais, glândulas salivares, de pele não melanoma, melanoma de pele, de cabeça, pescoço, lábios, orbitários, craniofacial, laringe, esvaziamento cervical, tireoide e outros.

A discussão do tema foi proposta entre os residentes durante a permanência na clínica de cabeça e pescoço do referido hospital que durou um mês e em que foi possível observar fragilidades e disparidades em relação a classificação dos diagnósticos de enfermagem e das características definidoras dos diagnósticos de enfermagem propostos pela equipe de enfermeiros da clínica, para isso foi utilizado a metodologia de diagnóstico situacional ou organizacional.

O diagnóstico situacional ou organizacional é o resultado de um processo de coleta, tratamento e análise dos dados colhidos no ambiente trabalhado, sendo uma importante ferramenta utilizada no âmbito da gestão, constituído por "pesquisa” oriundos da participação efetiva das pessoas que atuam no local de estudo, para posteriormente planejar e programar ações ${ }^{(11)}$.

A partir deste diagnóstico situacional, foi proposta a elaboração de um protocolo assistencial para pacientes com câncer de cabeça e pescoço, segundo os diagnósticos de enfermagem prevalentes na clínica, sendo desenvolvido no mês de abril de 2021, objetivando elaborar um método eficiente, prático e de fácil manuseio. 
A elaboração do produto se deu mediante quatro etapas, sendo a etapa 1 (Diagnostico situacional), etapa 2 (levantamento de dados clínicos dos pacientes internados), etapa 3 (levantamento de dados clínicos de pacientes com CCP encontrados na literatura), etapa 4 (Desenvolvimento do Protocolo).

A etapa 1 ocorreu mediante a realização de um diagnóstico situacional das possíveis problemáticas encontradas na clínica, durante a socialização das fragilidades identificadas, os pesquisadores definiram como um ponto de necessidade a instituição de um protocolo assistencial, o qual definisse os diagnósticos de enfermagem (segundo a classificação NANDA-I) para a assistência perioperatória de pessoas com câncer de cabeça e pescoço. A etapa 2, constituiu-se da caracterização das necessidades assistenciais dos pacientes internados na clínica de cabeça e pescoço, segundo a etapa de coleta de dados do Processo de Enfermagem, realizada durante a admissão desses pacientes e das visitas de rotina.

A etapa 3, constitui-se do levantamento das evidências científicas sobre as necessidades humanas básicas de pessoas com câncer de cabeça e pescoço durante o período perioperatório. Para tanto, foram incluídos no estudo: artigos completos e disponíveis gratuitamente nas bases de dados utilizadas, disponíveis no idioma português. Foram excluídos editoriais, cartas ao editor, resumos, opinião de especialistas e revisões. https://doi.org/10.31011/reaid-2021-v.95-n.36-art.1129 Rev Enferm Atual In Derme v. 95, n. 36, 2021 e-021168
As informações extraídas dos artigos foram pautadas nos diagnósticos de enfermagem de pacientes de cirurgia de cabeça e pescoço. Os descritores utilizados para mediar a busca das evidências foram: Cuidados de Enfermagem; Diagnóstico de Enfermagem e Enfermagem e as palavra-chave associadas foram: cirurgia de cabeça e pescoço e neoplasia de cabeça pescoço.

Na quarta e última etapa, deu-se início ao processo de elaboração do protocolo, onde se realizou o agrupamento das informações extraídas em cada uma das etapas anteriores associadas com os diagnósticos da classificação NANDA-I (2018-2020), para o embasamento e elaboração da tecnologia ${ }^{(13)}$. Após isso, iniciou-se o processo de edição e diagramação, seguindo critérios de estrutura, organização, layout, linguagem, design, adequação funcional prática ao público específico, acerca dos principais diagnósticos de enfermagem identificados nos usuários internados na clínica de cabeça e pescoço do hospital em questão. O intuito do protocolo desenvolvido é de caracterizar-se como um instrumento de consulta rápida, haja vista que cada paciente apresenta sinais e sintomas específicos, ainda que a doença de base seja a mesma, sendo necessário a consulta ao exemplar completo para buscar a descrição do diagnóstico encontrado que não esteja no produto criado pelos autores.

A experiência foi desenhada a partir da observação direta dos pesquisadores em seu ambiente de internato sem nenhuma 4 
anotação ou registro. Dessa forma, informa-se que este estudo, por se constituir em um relato de experiência, não sendo necessária à avaliação do Comitê de Ética em Pesquisa. No entanto, durante o seu desenvolvimento, ressalta-se que foram considerados os preceitos éticos da Resolução n. ${ }^{\circ} 466 / 12.8$, esclarecendo que não foram utilizados dados de pacientes ou de seus prontuários no estudo.

\section{RESULTADOS}

O internato na Clínica Cabeça e Pescoço se deu no mês de abril de 2021. A ideia surgiu após uma análise utilizando a metodologia de diagnóstico situacional, baseados nos problemas potenciais identificados no serviço de enfermagem, que foram definidos como o ponto chave de intervenção e implementação.

Foi possível observar fragilidades em relação a realização satisfatória dos diagnósticos de enfermagem pela equipe de enfermeiros da clínica, seja pela desatualização, falta de conhecimento ao manusear o NANDA ou incompatibilidade em desenvolver a sistematização da assistência de enfermagem e aplicar os diagnósticos de enfermagem no referido setor, conforme os problemas potencias que se observam nos usuários ali internados.
Diante disso, objetivando solucionar esse problema, foi produzido um material e impresso em folhas de papel A4, encadernado, em formado retrato, dividido em domínios, conforme o exemplar NANDA-I, o qual serviu como referência e embasamento para o desenvolvimento do produto em questão. Na capa do impresso consta a apresentação do informativo, intitulado: "DIAGNÓSTICO DE ENFERMAGEM DA CLÍNICA CABEÇA E PESCOÇO”, além da identificação e emblema do referido hospital em que o impresso fora implementado e emblema das UEPA e CESUPA, instituições as quais os autores são filiados.

Os tópicos foram definidos a partir de um diagnóstico situacional, embasados nos problemas potenciais identificados nos usuários internados na clínica, que foram definidos como o ponto chave de intervenção e implementação. Os itens que entraram para o conteúdo do impresso foram: I) Promoção da saúde, II) Nutrição III) Eliminação e Troca, IV) Atividade e repouso, V) Percepção/Cognição, VI) Auto-percepção, VII) papeis e relacionamento, VIII) Enfrentamento / tolerância ao estresse, IX) Segurança/proteção e X) Conforto. Os diagnósticos de enfermagem que compõem cada domínio estão dispostos no Quadro I. 
Quadro 1 - Diagnósticos de enfermagem mais prevalentes da clínica Cabeça e Pescoço, HOL, Belém, PA, 2021.

\begin{tabular}{|c|c|}
\hline DOMÍNIO & DIAGNÓSTICO DE ENFERMAGEM \\
\hline I - Promoção da saúde & $\begin{array}{l}\text { Envolvimento em atividades de recreação diminuído/ Estilo de } \\
\text { vida sedentário/ Manutenção ineficaz da saúde/ Síndrome do } \\
\text { idoso frágil }\end{array}$ \\
\hline II - Nutrição & $\begin{array}{l}\text { Deglutição prejudicada/ Nutrição desequilibrada: menor do que } \\
\text { as necessidades corporais/ Obesidade/ Sobrepeso/ Volume de } \\
\text { líquidos deficiente }\end{array}$ \\
\hline III - Eliminação e Troca & Constipação/ Diarreia/ Motilidade gastrintestinal disfuncional \\
\hline IV-Atividade e Repouso & $\begin{array}{l}\text { Distúrbio no padrão de sono/ Privação de sono/ Padrão } \\
\text { respiratório ineficaz }\end{array}$ \\
\hline V - Percepção/Cognição & Conhecimento deficiente/ Comunicação verbal prejudicada \\
\hline VI-Auto-percepção & $\begin{array}{l}\text { Risco de dignidade humana comprometida/ Distúrbio na } \\
\text { identidade pessoal/ Baixa autoestima situacional }\end{array}$ \\
\hline $\begin{array}{l}\text { VII - Papeis e } \\
\text { relacionamento }\end{array}$ & Interação social prejudicada \\
\hline $\begin{array}{l}\text { VIII - } \\
\text { Enfrentamento/tolerância } \\
\text { ao estresse }\end{array}$ & $\begin{array}{l}\text { Síndrome do estresse por mudança/ Ansiedade/ Medo/ } \\
\text { Sentimento de impotência/ Regulação do humor prejudicada }\end{array}$ \\
\hline IX-Segurança e Proteção & $\begin{array}{l}\text { Risco de infecção/ Risco de aspiração/ Desobstrução ineficaz } \\
\text { de vias aéreas/ Integridade da membrana mucosa oral } \\
\text { prejudicada/ Integridade da pele prejudicada/ Integridade tissular } \\
\text { prejudicada/ Risco de lesão na córnea/ Risco de Recuperação } \\
\text { cirúrgica retardada/ Risco de sufocação/ Risco de sangramento/ } \\
\text { Risco de reação adversa a meio de contraste iodado/ } \\
\text { Hipertermia/ Hipotermia/ Risco de hipotermia perioperatória }\end{array}$ \\
\hline $\mathrm{X}$ - Conforto & Conforto prejudicado/ Dor aguda/ Dor crônica/ Náuseas \\
\hline
\end{tabular}

Fonte: NANDA-I, 2018

Objetivou-se apresentar o conteúdo do impresso de forma clara, concisa e objetiva, considerando a praticidade do acesso e a limitação física, visto que o instrumento é uma ferramenta de consulta breve, pautado em evidências científicas, adaptada para a realidade do hospital onde este estudo foi https://doi.org/10.31011/reaid-2021-v.95-n.36-art.1129 Rev Enferm Atual In Derme v. 95, n. 36, 2021 e-021168 desenvolvido e para atender as necessidades de pessoas com CCP durante a internação até o momento de alta hospitalar, além disso, o protocolo propiciará aos enfermeiros do setor viabilizar a assistência em saúde prestada aos usuários. 
No design, foram aplicados poucos recursos visuais, apenas dividindo os domínios dos diagnósticos por cor e quanto as classes dos diagnósticos, propriamente ditos, foram colocadas em fontes garrafais $\mathrm{e}$ destacadas em negrito, a fim de tornar o informativo mais interessante e acessível, com uma busca sem mais esforços, porém sem comprometer o conteúdo do instrumento.

No final do impresso, fora inserido um $Q R$ code, com um link direto para o NANDAI (2018-2021), caso os enfermeiros do local sintam a necessidade de incluir algum diagnóstico a mais, segundo outras necessidades de cuidado do usuário. Este protocolo visa contribuir com a assistência perioperatória a pessoas com CCP internadas nesta clínica, e com o planejamento da SAE dos enfermeiros do serviço.

Após a disponibilização do produto tecnológico, os residentes buscaram a avaliação dos enfermeiros da clínica em relação a tecnologia proposta, durante a discussão e avaliação do produto, percebeu-se que o domínio IX) Segurança/Proteção foi o mais abordado e o que os enfermeiros relatam haver mais necessidade de ser implementado, haja vista que a maioria dos diagnósticos presentes neste domínio, tais como respiração, deglutição e comunicação verbal foram destacados como de maior complexidade de enfrentamento pelos resultados encontrados na revisão de literatura, pelos relatos dos enfermeiros do setor, bem como pela observação do cenário de prática dos residentes.

Explorar e buscar desenvolver tecnologias cuidativo-assistenciais embasadas em evidências científicas são fundamentais para a práxis de enfermagem, e para a reabilitação da saúde de um indivíduo. Ao desenvolver esta tecnologia pudemos ser autores do processo de facilitação da rotina e de educação continuada dos enfermeiros da clínica de câncer de cabeça e pescoço em relação ao planejamento da assistência perioperatória de pessoas com CCP.

Por fim, o instrumento foi enviado para a comissão de risco do referido hospital para que fosse avaliada a sua eficácia, real necessidade perante a clínica, assim como a precisão dos enfermeiros por este produto, além de também avaliar o instrumento como um mecanismo que pode ser incorporado e utilizado em larga escala na clínica sem comprometer o fluxo do serviço ou gerar viés e maleficência aos usuários e desconformidade com a assistência de enfermagem prestada no setor.

\section{DISCUSSÃO}

O desenvolvimento de um instrumento para facilitar o acesso e a abordagem as demandas dos pacientes, baseado na observação diária e na realidade do serviço, propiciou aos enfermeiros e residentes conhecer e reunir os principais diagnósticos de enfermagem apresentados pelos usuários, colaborando para o processo de enfermagem 
realizado pelos profissionais. Conhecer os principais DE dos pacientes, permite uma comunicação padronizada, possibilitada pelo conhecimento do perfil diagnóstico, além de melhorar o desenvolvimento do PE, com a possibilidade de intervenções individualizadas e ações gerenciais com qualidade ${ }^{(14)}$.

Identificou-se, durante as visitas de enfermagem e pelo próprio convívio com os usuários da clínica, que o item IX) Segurança e Proteção, descrito neste estudo, foi $o$ domínio do NANDA-I que mais se observou nos clientes internados. Descreve-se que o CCP afeta e altera funções básicas e essenciais para um bom convívio no cotidiano, incluindo segurança e proteção, como a respiração, deglutição e comunicação verbal, além de comprometer o funcionamento fisiológico de algumas ações, como a mastigação, disfagia, aspiração, alteração na fala e na estética ${ }^{(15,16)}$.

Alterações em funções essenciais como a capacidade de mastigar, de se comunicar e interagir socialmente, podem ser fatores preponderantes de sofrimento, pois pode levar a disfunções importantes na vida diária, e isso se dá pela própria localização anatômica do CCP. Além disso, é muito comum a ocorrência de alteração de humor quando avaliado os DE desses usuários, sendo a ansiedade e a depressão sintomas bem comuns desde o diagnóstico médico ${ }^{(17)}$.

As alterações provocadas pelo $\mathrm{CCP}$, atreladas aos efeitos colaterais do tratamento, podem, muitas vezes, levar o paciente a experimentar a sensação de desesperança com a situação vivenciada; uma revisão sistemática com meta-análise encontrou evidências de que a intervenção de enfermagem tem um efeito positivo no sentimento de esperança ${ }^{(18)}$. Assim, acreditamos que intervenções complementares de enfermagem e o DE eficaz auxiliam nos aspectos de segurança e proteção do paciente com CCP.

A taxonomia de enfermagem que sustentou o desenvolvimento desta pesquisa foi a NANDA-I, que oferece uma comunicação reconhecida de forma internacional e de modo universal pelos enfermeiros, que além de propiciar parâmetros fidedignos para a identificação de DE, possibilita a redução de complicações clínicas e o controle de riscos, fortalecendo, dessa forma, a enfermagem como ciência pautada em evidências ${ }^{(19)}$.

Nos resultados deste estudo também é descrito a respeito da fragilidade em desenvolver os DE na enfermaria em questão, sendo este um dos motivos para a dissertação desta pesquisa. Infere-se que os profissionais de enfermagem precisam desenvolver habilidades técnicas-científicas para investigar, diagnosticar, planejar, implementar e avaliar o usuário levando em consideração as evidências, somadas ao processo holístico, para a implementação de um diagnóstico correto, tendo em vista a 
melhor solução para os problemas identificados $^{(20)}$.

Assim, ressalta-se que o desenvolvimento do DE é uma etapa fundamental do PE que é realizado pelo enfermeiro, pois redireciona as demais etapas do PE e promove um melhor planejamento da assistência e reconhecimento do trabalho da enfermagem, além de promover a atuação com vista a atender as necessidades individuais de cada indivíduo ${ }^{(21)}$.

Reforça-se ainda que o DE é parte fundamental do PE e que por sua vez influencia na SAE desenvolvida pelo enfermeiro, porém se observa que o desenvolvimento desta ação esbarra em dificuldades como a falta de capacitação dos enfermeiros e o próprio desconhecimento dos profissionais, seja pela limitação da funcionalidade desta ação, pela falta de recursos humanos e materiais ou pelo baixo apoio institucional e gerencial em relação a esta prática ${ }^{(22)}$.

Reforça-se que a dificuldade de estruturar a SAE também é reforçada pela baixa remuneração que os profissionais da enfermagem recebem em contraste com a intensa e longa jornada de trabalho, pelo cansaço físico e mental referido pela classe e as complicadas relações sociais advindas do próprio ambiente hospitalar, sem contar a desvalorização e falta de reconhecimento da prescrição de enfermagem para dar resolutividade ao DE pelos demais membros da equipe ${ }^{(23)}$. 
promover melhores cuidados e qualidade de vida para os clientes.

Considera-se a aplicação de tecnologias educativas em saúde como ferramenta primordial para facilitar $o$ entendimento, e o seu uso deve ser constante pelos profissionais de saúde ${ }^{(27)}$. No entanto, se faz necessários outros estudos que abordem o cuidado ao paciente perioperatório de cirurgia de Cabeça e Pescoço.

\section{CONSIDERAÇÕES FINAIS}

O estabelecimento de diagnósticos de enfermagem perpassa por um processo crítico, reflexivo que demanda conhecimento com base científica dos profissionais, que impactam na qualidade de vida que os usuários terão, sobretudo conferindo qualidade à assistência prestada e consequentemente satisfação aos usuários com as ações e serviços que são desenvolvidos.

A elaboração do produto sobre os principais diagnósticos na clinica de cabeça e pescoço do referido hospital, pelos residentes, demonstrou-se como uma estratégia de enorme potencial para orientação e organização das ações e serviços da enfermagem no desenvolvimento do cuidado integral a esse perfil de usuário tão peculiar, além de ter possibilitado a discussão sobre a temática entre a equipe de enfermeiros, melhorando a percepção dos profissionais ao que fora exposto, despertando a sensibilidade da classe a respeito das dificuldades encontradas e um maior envolvimento e conhecimento dos mesmos acerca do DE e sua importância para a pratica clínica.

Para os autores, esta experiencia foi se suma importância, pois contribuiu para o fortalecimento de uma visão abrangente e perceptiva sobre as diversas nuances do indivíduo, permitindo classificar as respostas humanas utilizando como base uma taxonomia ordenando tópicos em categorias, além de consolidar um olhar cientifico e holístico em cima de uma problemática, que foi identificada e sofreu teorização para desenvolvimento das hipóteses para desvelar o motivo do problema, além da avaliação da intervenção sobre o ponto-chave, conferindo um fazer mais sensível e humanizado, ético, com respeito e reconhecimento pelas ações desenvolvidas pela equipe de enfermagem.

Para os enfermeiros da clínica que receberam o produto, a elaboração e implementação do material serviu como uma ferramenta mais para auxiliar no desenvolvimento do cuidado, além disso, o envolvimento dos profissionais se mostrou relevante e ativo durante todo o processo, visto que ficou evidente que será um atributo a mais para organizar a assistência.

E por fim, é primordial destacar a necessidade de se estimular novos estudos que relacione a Sistematização da Assistência de Enfermagem (SAE) e o desenvolvimento de conhecimentos referentes às intervenções de enfermagem na área de cirurgia de cabeça https://doi.org/10.31011/reaid-2021-v.95-n.36-art.1129 Rev Enferm Atual In Derme v. 95, n. 36, 2021 e-021168 
e pescoço, haja vista que esse é um perfil de pacientes pouco explorado pela enfermagem.

\section{REFERÊNCIAS}

1. da Silva FA, Roussenq SC, Tavares MGDS, Souza CPFD, Mozzini CB, Benetti M, Dias M. Perfil Epidemiológico dos Pacientes com Câncer de Cabeça e Pescoço em um Centro Oncológico no Sul do Brasil. Revista Brasileira De Cancerologia. 2020; 66(1), e-08455. https://doi.org/10.32635/21769745.RBC.2020v66n1.455.

2. Niero AC, Salvaro MS, Hoepers NJ, Zugno PI. Cuidados de enfermagem ao paciente oncológico em pósoperatório de cirurgia de cabeça e pescoço. Revista Interdisciplinar de Estudos em Saúde. 2018; 249-262.

3. Majid A, Sayeed BZ, Khan M, Lakhani M, Saleem MM, Rajani H, et al. Assessment and Improvement of Quality of Life in Patients Undergoing Treatment for Head and Neck Cancer. Cureus. 2017; 9(5):e1215. doi: http://doi.org/10.7759/cureus.121 $\underline{5}$

4. Hortense FTP, Bergerot, CD, Domenico, EBiLD. Qualidade de vida, ansiedade e depressão de pacientes com câncer de cabeça e pescoço: estudo clínico randomizado. Revista da Escola de Enfermagem da USP. 2020; 54, e03546. https://doi.org/10.1590/s1980220x2018040103546.

5. Brasil. Ministério da Saúde. Instituto Nacional de Câncer José Alencar Gomes da Silva. Estimativa 2018: incidência de câncer no Brasil Internet. Rio de Janeiro: INCA; 2017 Acesso em: 05 de abril de 2021. Disponível em: https://www.inca.gov.br/publicacoes/li vros/estimativa-2018-incidencia-decancer-no-brasil

6. Pinto GP, Mont'alverne DGB. Neoplasms of head and neck: impacts functional and quality of life. Rev. Bras. Cir Cabeça Pescoço. 2015;44(3):152-6. Available from: http://www.sbccp.org.br/wpcontent/uploads/2015/07/Rev-SBCCP44-3-artogo-09.pdf.

7. NANDA-I, Inc. Diagnósticos de enfermagem da NANDA: 2015-2017. Porto Alegre: Artmed; 2015.

8. Horta WA. Processo de enfermagem. São Paulo: EPU 1979; 99p.

9. Mendes FA, da Silva, MP, Ferreira, CR Salles. Diagnósticos de enfermagem em portadores de hipertensão arterial na atenção primária. Estação Científica (UNIFAP). 2018; v. 8, n. 1, p. 91-101.

10. Silva MR, Silva DO, Santos EC, Oliveira PP, Sales AS, Rodrigues AB. Diagnoses, results and nursing interventions for people submitted to orthopedic and traumatological surgeries. Rev enferm UFPE online. 2017; 11(5):2033-45. Acesso em 23 de mai 2021. doi: http://dx.doi.org/10.5205/reuol.930281402-1-RV.1105sup201708.

11. Sant'anna CF, Cezar-Vaz MR, Cardoso LS. Comunidade: objeto coletivo do trabalho das enfermeiras da Estratégia Saúde da Família. Acta Paul Enferm. 2011; 24(3):341-47.

12. de Oliveira LG, Abreu AS, Nascimento FS, Pinheiro MLB, Sousa AM, Nascimento BYB, et al. Diagnósticos e intervenções de enfermagem à paciente idosa com tuberculose em unidade básica de saúde: relato de experiência. Brazilian Journal of Health Review. 20219; 2(1), 580-89. 
13. Conselho Federal de Enfermagem. Resolução COFEN no 358/2009. Sistematização da Assistência de Enfermagem e a implementação do Processo de Enfermagem em ambientes, públicos ou privados, em que ocorre o cuidado profissional de Enfermagem [Internet].. Disponível em:

http://www.portalcofen.gov.br/Site/20 07/materias.

asp?ArticleID=10113§ionID=34.

Acesso em: 18 de mai 2021.

14. Rodrigues AB, Aguiar MIFD, Oliveira PPD, Ferreira IS, Magalhães TDL, Barbosa JEC. Diagnósticos de enfermagem em pacientes com câncer de cabeça e pescoço em tratamento ambulatorial / Nursing diagnosis in patients with head and neck cancer in ambulatorial treatment. Ciência, Cuidado E Saúde. 2019; 18(4). https://doi.org/10.4025/cienccuidsaude .v18i4.45510.

15. Avelar JMDP, Nicolussi AC, Toneti BF, Sonobe HM, Sawada NO. Fadiga em pacientes com câncer de cabeça e pescoço em tratamento radioterápico: estudo prospectivo. Revista LatinoAmericana de Enfermagem. 2019; 27.

16. Reis JB, Oliveira JM, Nascimento VF, Cabral JF, Lucietto GC, Silva RA. Câncer de cabeça e pescoço: a comunicação e os seus significados. Revista de Enfermagem UFPE on line. 2018; 12(12), 3263-70. doi:https://doi.org/10.5205/19818963-v12i12a237730p3263-32702018.

17. Rodrigues $\mathrm{AB}$, Cunha $\mathrm{GH}$, Aquino CBQ, Rocha SR, Mendes CRS, Firmeza MA, Grangeiro ASM. Câncer de cabeça e pescoço: validação de instrumento para coleta de dados. Revista Brasileira de Enfermagem. 2018; 71(4), 1899-906. https://doi.org/10.1590/0034-7167$\underline{2017-0227}$
18. Li P, Guo YJ, Tang Q, Yang L. Effectiveness of nursing intervention for increasing hope in patients with cancer: a metaanalysis. Rev. LatinoAm. Enfermagem. 2018; 26:e2937. doi: http://dx.doi.org/10.1590/15188345.1920.2937

19. Santana ET, Coutinho GG, Silva DVA, Bernardes TAA, Camisasca LR, Gusmão ROM, Araújo DD. Diagnósticos de enfermagem da taxonomia NANDA-I para idosos em instituição de longa permanência. Escola Anna Nery. 2021; 25(1), e20200104. Epub August 10, 2020.https://doi.org/10.1590/21779465-ean-2020-0104

20. Facione PA, Crossetti MGO, Riegel F. Pensamento Crítico Holístico no Processo Diagnóstico de Enfermagem. Revista Gaúcha de Enfermagem. 2017; 38(3), e75576. Epub April 09, 2018.https://doi.org/10.1590/19831447.2017.03.75576

21. Debone MC, Pedruncci ESN, Candido MCP, Marques S, Kusumota L. Diagnósticos de enfermagem em idosos com doença renal crônica em hemodiálise. Revista Brasileira de Enfermagem. 2017; 70(4), 800-805. https://doi.org/10.1590/0034-71672017-0117.

22. Gome RMGM, Teixeira LSTS, dos Santos MDCQ, dos Santos Q, Sales ZNSN, Linhares EFLF, Santos KASA. Sistematização da assistência de enfermagem: revisitando a literatura brasileira. ID On Line Revista De Psicologia. 2018; 12(40), 995-1012.

23. Nunes RM, Nunes MR, Assunção IA, Souza LL. Sistematização da assistência de enfermagem e os desafios para sua implantação na unidade de terapia intensiva: uma revisão de literatura. Revista Uningá. 2019; 56(S2), 80-93. 
24. Silva GN, Santos GL, Silva CJ, Lima FC, Ueno TMRL, Moura LDO, Marcena JC, Campos FLM, Oliveira VMLP, Ferreira MFDC, Fonseca ACM, Barral DM, Ramalho MA. The nurse's perception about the systematization of nursing care to premature newborn in the intensive care unit. Research, Society and Development. 2021; 10(3), e16510313119.

https://doi.org/10.33448/rsd-

$\underline{\text { v10i3.13119 }}$

25. Herdman TH, Kamitsuru S. Diagnósticos de enfermagem da NANDA: definições e classificação 2015-2017. 2015

26. Riegel F, Oliveira Junior, NJ. Processo de enfermagem: implicações para a segurança do paciente em centro cirúrgico. Cogitare Enfermagem. $2017 ; 22(1)$.
27. Costa LKC, Cardoso BCR, Oliveira LG, Palheta AMS, Luz VCS, Aviz SPC, et al. Uso de tecnologia leve para o esclarecimento sobre o exame citopatológico de usuárias atendidas em uma Unidade Básica de Saúde no Município de Ananindeua, Pará, Brasil. Revista Científica Multidisciplinar Núcleo do Conhecimento. 2020; 05, 06, Vol.08, pp. 49-57. DOI: 10.32749/nucleodoconhecimento.com. $\mathrm{br} /$ saude/exame-citopatologico

\section{Autor correspondente \\ Letícia Gomes de Oliveira \\ Endereço: Conj. Saint Clair Passarinho, n. 22 - CEP 67125-570 \\ Contato: +55 (91) 98489-3640 \\ E-mail: gomes_15_letici@hotmail.com}

Submissão: 2021-05-30

Aprovado: 2021-11-08 